\title{
Redução dos Níveis de Corrupção no Brasil: Qual o papel da Tecnologia da Informação e Comunicação?
}

\section{Reduction of Corruption Levels in Brazil: What is the role of Information and Communication Technology?}

Odirlei Antonio Magnagnagno ${ }^{1}$, Edimara Mezzomo Luciano ${ }^{1}$, Guilherme Costa Wiedenhöft ${ }^{1}$

\author{
${ }^{1}$ Pontifícia Universidade Católica do Rio Grande do Sul, PUCRS, Brasil \\ Correspondência: Odirlei Antonio Magnagnagno. Endereço: Av. das Torres, 500 FAG 85806-095 - Cascavel, PR \\ - Brasil Telefone: (045) 33213900 E-mail: odirlei@fag.edu.br
}

Recebido: 04 de dezembro de 2017 Aceito: 15 de dezembro de 2017 Publicado: 31 de dezembro de 2017

DOI: http://dx.doi.org/10.21714/1679-18272017v15Ed.p157-170

\begin{abstract}
Resumo
O principal objetivo do artigo é identificar como aplicações de TICs têm sido utilizadas para reduzir os níveis de corrupção. Inicialmente, as causas da corrupção no Brasil foram identificadas em publicações sobre o tema, por meio de uma revisão sistemática da literatura. Da mesma forma, artigos sobre TIC e corrupção foram analisados. As duas rodadas de revisão sistemática de literatura seguiram um protocolo de pesquisa formalizado para responder a seguinte questão de pesquisa: quais podem ser as contribuições da TIC na redução dos níveis de corrupção? O estudo é exploratório com uma abordagem qualitativa. A partir da análise detalhada dos artigos por meio de uma análise de conteúdo categorial, 10 causas da corrupção no Brasil foram identificadas, além de seis aplicações práticas de TIC que posteriormente foram discutidas conjuntamente. A principal contribuição teórica do artigo é relacionar as aplicações de TIC com as causas da corrupção, embasando a discussão de como a utilização da TIC pode atuar para evitar ou atenuar os impactos da corrupção. Como contribuições práticas, representantes de órgãos governamentais podem utilizar o resultado desta pesquisa para aplicar práticas de TIC $\mathrm{e}$ melhorar a governança pública em seus esforços para a redução dos níveis de corrupção.
\end{abstract}

Palavras-chave: Corrupção, causas da corrupção, TIC, organizações públicas, governança eletrônica.

\begin{abstract}
The main purpose of this article is to identify how ICT applications have been used to reduce levels of corruption. The causes of corruption in Brazil were identified in previous publications through a systematic literature review as well as ICT and corruption. The two rounds of systematic literature review followed a formalized research protocol in order to answer the following research question: what can the ICT contributions to reduce the levels of corruption be? The data analysis on this exploratory and qualitative study was performed through a categorial content analysis. Based on that 10 causes of corruption in Brazil were identified, as well as six practical ICT applications that were later discussed together. The main theoretical contribution of this paper is to connect ICT applications to the causes of corruption, supporting the discussion on how ICT can be used to avoid or mitigate the impacts of corruption. As a practical contribution, representatives of government agencies can use the results of this research to apply ICT practices and improve public governance in their efforts to reduce levels of corruption.
\end{abstract}

Keywords: Corruption, causes of corruption, ICT, public organizations, electronic governance.

Esta obra está licenciada sob uma Licença Creative Commons Attribution 3.0.

\section{Introdução}

Como decorrência dos movimentos por reforma do estado, accountability e prestação de contas, e também o crescimento do uso de dispositivos de TIC pela população, surge a discussão por maiores e melhores níveis de transparência por parte das organizações públicas. Iniciando como um desejo e se configurando em pressão por parte dos stakeholders, a transparência, quando aplicada à gestão pública, vai muito além de dar a conhecer à 
população números sobre a atuação da gestão de uma organização pública. Segundo Fox (2007), a transparência se constitui em uma regra de conduta a ser seguida pelos agentes públicos, de forma que suas ações sejam abertas à sociedade e possibilitem o controle social por parte dos cidadãos.

Na medida em que a discussão evolui, é possível observar uma mudança gradativa do foco de utilização de recursos de TIC, de ferramentas que incrementam a conveniência da prestação de serviços do próprio governo para tornar-se também um facilitador da reforma administrativa e um promotor da participação democrática (YILDIZ, 2007).

A participação, associada ao uso estratégico de TIC, criou um novo modelo de administração pública, o chamado Governo Eletrônico (AGUNE; CARLOS, 2005). A Organization for Economic Cooperation and Development (OECD, 2003) considera que Governo Eletrônico envolve as seguintes perspectivas: a) a entrega de serviços ao cidadão através da internet e outras atividades baseadas na internet desenvolvidas por parte do governo; b) outros usos das TICs pelo governo que não serviços eletrônicos; c) A transformação da administração pública através do uso das TICs. Esses conceitos buscam aumentar a participação do cidadão e proporcionar a transparência à administração pública.

Estudos como o de Jaeger e Bertot (2010) têm abordado a questão da transparência como uma fonte primária de relação do cidadão com o estado. Muito embora a transparência possa ser feita utilizando diferentes meios, é com a utilização das TICs que a transparência de mais dados a mais cidadãos começa a se tornar possível. O Brasil tem feito avanços significativos em termos de uso de TIC no governo, mas estes ainda não têm impactado na redução dos níveis de corrupção. Isso é o que mostra a pesquisa Corruption Perception Index, organizada pela Transparency International e a ONU desde 2001. Neste ranking, que analisa um total de 176 países e na qual a posição de número um é a do país com o menor nível de corrupção, o Brasil ocupa a posição número 79 (TRANSPARENCY INTERNACIONAL, 2016). Essa é uma posição intermediária, considerando o conjunto de países analisados. No entanto, o Brasil tem perdido posições nos últimos anos, o que indica que na percepção dos respondentes, os níveis de corrupção aumentaram.

A literatura apresenta abordagens distintas sobre corrupção, em especial a legal, econômica, política, cultural e administrativa. A abordagem legal é vista sob três frentes, sendo a primeira a má aplicação das leis (SPECK, 2003), a segunda pela falta delas (KÜHL TELES, 2007) e a terceira pela criação de leis que contribuam com a corrução (DIAS; BENTO, 2011). A abordagem econômica discorre a respeito dos prejuízos econômicos à sociedade, os quais envolvem principalmente a obtenção de vantagens financeiras ilegais por parte dos agentes envolvidos (DEL MONTE; PAPAGNI, 2001). A dimensão política pode ser exemplificada com a compra de votos (SPECK, 2003) e o nepotismo (PASCARELLI FILHO, 2013). A dimensão cultural trazida por Power e González (2003) aborda a questão de acordo com a região ou país, e procura entender se de fato os atributos culturais podem explicar ao menos uma parte da variação no nível de corrupção entre e dentro das regiões do mundo. Entre os estudos acerca da dimensão administrativa, Melo, Sampaio e Oliveira (2015) mostram que a abertura de empresas nos estados brasileiros é afetada negativamente pela incidência da corrupção. Cada uma dessas abordagens têm diferentes entendimentos sobre quais são as causas da corrupção e quais possíveis maneiras de reduzir a sua ocorrência ou intensidade.

Este estudo aborda a análise da corrupção em organizações públicas e tem como foco a dimensão administrativa, a qual apresenta potencial de reduzir os níveis de corrupção em especial pela ampliação da capacidade de gestão e da eficiência, e pela melhoria institucional da gestão pública (FILGUEIRAS; ARANHA, 2011), bem como pela governança eletrônica e controle social. Para este estudo, adota-se a definição de Jain (2001) para corrupção, que a define como sendo os atos nos quais o poder de servidores públicos é utilizado para ganhos pessoais de forma que viola as regras vigentes. Estas regras podem ser tanto a legislação como o que é considerado adequado em um determinado grupo populacional, de acordo com os aspectos culturais. Estes atos podem assumir muitas formas, incluindo suborno, peculato, roubo, extorsão, abuso de poder, favoritismo, explorando conflitos de interesses e ausência de políticas suficientes para coibi-lo (UNODC, 2004).

Novos modelos para o relacionamento Estado/sociedade surgiram com a evolução da TI, dos meios de comunicação e particularmente da Internet (GUIMARÃES; MEDEIROS, 2005). Para Cunha e Miranda (2013), os avanços da TIC geraram oportunidades para transformar o relacionamento entre o governo e os cidadãos, contribuindo para alcançar os objetivos da boa governança. Nesse sentido, é importante destacar os esforços do chamado Governo Eletrônico, visando melhores serviços ao cidadão, e aqueles em busca de transparência, dados e governo aberto e participação, utilizando as TICs como suporte (SRIVASTAVA; TEO; DEVARAJ, 2016). Ainda, a TIC pode contribuir por meio do monitoramento e da rastreabilidade das atividades dos funcionários do 
governo de uma maneira mais efetiva (SHIM; EOM, 2008). No entanto, a redução dos níveis de corrupção em um país é uma longa jornada construída dia a dia por muitas pessoas e iniciativas, e acredita-se que a utilização da TIC pode potencializar estes esforços no que tange ao escopo de gestão e governança. Assim, assume-se nesta pesquisa que a TIC pode ter um papel importante na contribuição para a redução dos níveis de corrupção.

O foco da pesquisa é identificar aplicações da TIC com algum tipo de contribuição na redução dos índices de corrupção, conforme pesquisas anteriores sobre o uso de TICs no governo. Em termos de problemática de estudo, esta pesquisa considera que é necessário investigar quais linhas de ação podem ser definidas para a redução dos níveis de corrupção utilizando as TICs como um meio de potencializar o resultado dos esforços em busca de transparência. Assim, busca-se responder por meio deste estudo a seguinte questão de pesquisa: que tipo de contribuição a TIC pode trazer para a redução dos níveis de corrupção em organizações públicas no Brasil? A relevância desta pesquisa sustenta-se em três pontos, quais sejam, a necessidade e importância da TIC para reduzir os níveis de corrupção, a relativamente baixa quantidade de produções sobre o tema no Brasil e a abordagem baseada em elementos de gestão e governança.

Considerando o fato de que para explorar soluções para um problema é preciso entender detalhadamente suas causas, considera-se fundamental identificar as origens da corrupção especificamente no contexto brasileiro. $\mathrm{O}$ objetivo do artigo é identificar as causas da corrupção no Brasil e as aplicações da TIC para lidar com estas causas, por meio de um levantamento sistemático de literatura, tendo como recorte a administração pública no Brasil. A partir das causas da corrupção é possível discutir o papel da TIC na redução da ocorrência ou intensidade das causas da corrupção.

A estrutura desta pesquisa seguiu a recomendação de Cooper, Hedges e Valentine (2009) para artigos baseados em revisão sistemática de literatura. Esta primeira seção discutiu o tema, o problema de pesquisa, o objetivo e a justificativa. A seção dois se dedica a explicar os procedimentos metodológicos. A seção três apresenta os resultados e as discussões da pesquisa, seguida das considerações finais, expostas na seção 4 .

\section{Procedimentos metodológicos}

A pesquisa tem um enfoque qualitativo, com uma abordagem exploratória e analisando dados secundários. A pesquisa exploratória, conforme Malhotra (2001) tem como principal objetivo proporcionar ao pesquisador uma maior familiaridade com o problema em estudo. A Revisão Sistemática de Literatura foi realizada em dois momentos e com objetivos distintos: primeiramente buscando identificar as causas da corrupção no Brasil e posteriormente as aplicações de TIC relacionadas à corrupção. A partir disso, foi possível discutir o papel da TIC associado à corrupção. A integração dos dois resultados é discutida na seção seguinte.

Cada momento da Revisão Sistemática da Literatura envolveu diversas atividades ou etapas, divididas em três fases, quais sejam, planejamento, realização da revisão e o relato da revisão, seguindo as recomendações de Brereton et al. (2007). De acordo com os autores, um fator de grande importância no desenvolvimento de um estudo baseado em dados secundários, como uma revisão sistemática da literatura, é o protocolo utilizado como base durante a fase de planejamento. Neste sentido, todas as etapas metodológicas utilizadas podem ser observadas no Quadro 1, a seguir.

Quadro 1: Procedimentos realizados na revisão sistemática da literatura

\begin{tabular}{|c|c|c|}
\hline Fase - Etapa - Passo & Procedimento - Causas da Corrupção & Procedimento - TIC e Corrupção \\
\hline $\begin{array}{l}1 \text {-1 -Especificação da } \\
\text { pergunta de pesquisa }\end{array}$ & $\begin{array}{l}\text { Quais são as causas da corrupção no } \\
\text { Brasil }\end{array}$ & $\begin{array}{l}\text { Contribuição da TIC no combate à } \\
\text { corrupção }\end{array}$ \\
\hline $\begin{array}{c}1-2 \text { - Protocolo de } \\
\text { pesquisa }\end{array}$ & $\begin{array}{l}\text { Busca de artigos científicos publicados } \\
\text { em periódicos revisados por pares e } \\
\text { indexados nas bases de dados } \\
\text { ProQuest, Sage, Scopus, Springer, Web } \\
\text { of Knowledge, Wiley, Emerald e } \\
\text { Scielo. Termos utilizados: corrupção e } \\
\text { Brasil ou Corruption e Brazil } \\
\text { (publicação em português ou inglês). } \\
\text { Período estipulado = Todos os anos (até } \\
\text { dezembro de } 2016 \text { ) }\end{array}$ & $\begin{array}{l}\text { Artigos publicados em português ou } \\
\text { inglês, considerando todos os anos de } \\
\text { publicação. Para o levantamento dos } \\
\text { artigos na literatura, foi realizada uma } \\
\text { busca na base de dados: Proquest e } \\
\text { Ebsco, Sage, Wos, Scielo e Scopus, } \\
\text { bases de dados multidisciplinares que } \\
\text { indexam periódicos de diversas áreas. } \\
\text { Considerando os termos TI ou IT and } \\
\text { (corruption ou corrupção). }\end{array}$ \\
\hline $\begin{array}{l}1-3 \text { - Validação do } \\
\text { protocolo de pesquisa }\end{array}$ & $\begin{array}{l}\text { A revisão do protocolo foi realizada } \\
\text { por outros dois pesquisadores }\end{array}$ & $\begin{array}{l}\text { A revisão do protocolo foi realizada } \\
\text { por um expert no assunto corrupção }\end{array}$ \\
\hline $\begin{array}{l}2-4 \text { - Identificação das } \\
\text { pesquisas relevantes }\end{array}$ & Retorno inicial de 358 artigos & Retorno inicial de 43 artigos \\
\hline
\end{tabular}




\begin{tabular}{|c|c|c|}
\hline Fase - Etapa - Passo & Procedimento - Causas da Corrupção & Procedimento - TIC e Corrupção \\
\hline $\begin{array}{l}2-5 \text { - Seleção dos } \\
\text { estudos primários }\end{array}$ & $\begin{array}{l}\text { Através da leitura dos títulos foi } \\
\text { realizada a retirada dos artigos } \\
\text { duplicados dentro de cada base de } \\
\text { dados e posteriormente entre as bases. } \\
\text { Exclusão de artigos em duplicidade por } \\
\text { terem entradas em diferentes idiomas. } \\
\text { Retirada dos artigos que claramente } \\
\text { não tratavam sobre corrupção ou sobre } \\
\text { o Brasil, restando } 114 \text { artigos. } \\
\text { Realizada a exclusão dos artigos que } \\
\text { tratavam exclusivamente do setor } \\
\text { privado, processada através da leitura } \\
\text { do título, resumo/abstract ou do artigo } \\
\text { todo, restando } 102 \text { artigos. }\end{array}$ & $\begin{array}{l}\text { Após a eliminação dos artigos } \\
\text { repetidos, restaram } 35 \text { artigos. Os } \\
\text { resumos destes artigos foram lidos e } \\
\text { com isso foram descartados } 5 \text { artigos, } \\
\text { por não trazerem em seu texto o } \\
\text { assunto pertinente à pesquisa. } \\
\text { Contudo, foram acrescentados outros } 7 \\
\text { artigos, que não estiveram na busca } \\
\text { inicial, porém continham citações e } \\
\text { assuntos pertinentes à pesquisa. Com } \\
\text { isso ficaram } 37 \text { artigos. }\end{array}$ \\
\hline $\begin{array}{l}2-6 \text { - Avaliação da } \\
\text { qualidade do estudo }\end{array}$ & $\begin{array}{l}\text { Seleção de somente } \\
\text { acadêmicos, descartando } 25 \text { artigos } \\
\text { referentes a artigos não acadêmicos ou } \\
\text { de conferências, capítulos de livros, } \\
\text { artigos em duplicidade ou com } \\
\text { abordagens superficiais, ficando } 87 \\
\text { artigos para a análise. }\end{array}$ & $\begin{array}{l}\text { Dos } 37 \text { artigos, } 8 \text { deles não eram } \\
\text { acadêmicos ou não eram revisados por } \\
\text { pares. Restando } 29 \text {. Deste restante, } 12 \\
\text { continham abordagens bem } \\
\text { superficiais, restando assim } 17 \text { artigos } \\
\text { para a análise. }\end{array}$ \\
\hline $\begin{array}{c}2 \text { - } 7 \text { - Extração dos } \\
\text { dados necessários }\end{array}$ & $\begin{array}{l}\text { Artigos organizados em uma planilha, } \\
\text { contemplando grandes grupos de } \\
\text { descrição, cobertura, âmbito, } \\
\text { abordagem do problema, causas, assim } \\
\text { como as informações básicas dos } \\
\text { artigos. }\end{array}$ & $\begin{array}{l}\text { Os artigos foram organizados em uma } \\
\text { planilha, extraindo de cada um deles: o } \\
\text { ano de publicação, a abordagem } \\
\text { metodológica, os autores, a revista, os } \\
\text { objetivos, os resultados, a classificação } \\
\text { Qualis, o tipo de TIC utilizado e como } \\
\text { ocorreu a aplicação da TIC. }\end{array}$ \\
\hline $2-8$ - Síntese dos dado & $\begin{array}{l}\text { Leitura detalhada de todos os artigos, } \\
\text { listando dados de identificação dos } \\
\text { artigos, dos periódicos e dos autores. } \\
\text { Análise de conteúdo categorial com } \\
\text { categorias de causas da corrupção } \\
\text { identificadas a posteriori. }\end{array}$ & $\begin{array}{l}\text { A caracterização dos dados dos artigos } \\
\text { ocorreu principalmente através do tipo } \\
\text { de TIC e da aplicação utilizada nos } \\
\text { estudos pesquisados. }\end{array}$ \\
\hline $\begin{array}{c}3-9 \text { - Escrever o } \\
\text { relatório }\end{array}$ & Seção de resultados deste artigo. & Seção de resultados deste artigo. \\
\hline $\begin{array}{c}3-10 \text { - Validação do } \\
\text { relatório }\end{array}$ & $\begin{array}{l}\text { A validação foi realizada por outros } \\
\text { dois pesquisadores, revisando todas as } \\
\text { etapas do modelo proposto. }\end{array}$ & $\begin{array}{l}\text { A validação foi realizada por outros } \\
\text { dois pesquisadores, revisando todas as } \\
\text { etapas do modelo proposto. }\end{array}$ \\
\hline
\end{tabular}

Fonte: Elaborado a partir de Brereton et al. (2007)

Após a execução de todas as etapas, os artigos resultantes de cada um dos procedimentos foram lidos detalhadamente para realizar a Análise do Conteúdo Categorial, seguindo especialmente as recomendações de Bardin (1977). As categorias foram identificadas a posteriori, ou seja, conforme foram surgindo na análise de cada artigo. A cada inclusão de uma nova categoria, os artigos anteriores eram analisados novamente, para verificar se citavam a categoria que fora incluída recentemente.

\section{Análise e Discussão dos Resultados}

Esta seção discorre sobre a análise dos artigos em termos de causas e características da corrupção e como a TIC pode contribuir.

\subsection{Causas da Corrupção}

O levantamento de dados apontou para 87 artigos referentes à corrupção no Brasil. Esses artigos foram publicados em 71 revistas diferentes, sendo 15 brasileiras e 56 internacionais (66 publicados em língua inglesa e 21 em língua portuguesa). Em se tratando de tipo de organização, 58 estudos abordam exclusivamente organizações públicas, 11 artigos são relativos a organizações públicas e privadas e 18 abordando aspectos supra organizacionais ou individuais. 
As publicações de estudos abordando as causas da corrupção no Brasil não apresentaram um grande volume ao longo das últimas quatro décadas, porém, tem crescido nos últimos anos. O pico de publicações foi entre $2012 \mathrm{e}$ 2015, conforme demonstrado na Figura 1.

Figura 1: Evolução anual das pesquisas sobre corrupção no setor público no Brasil

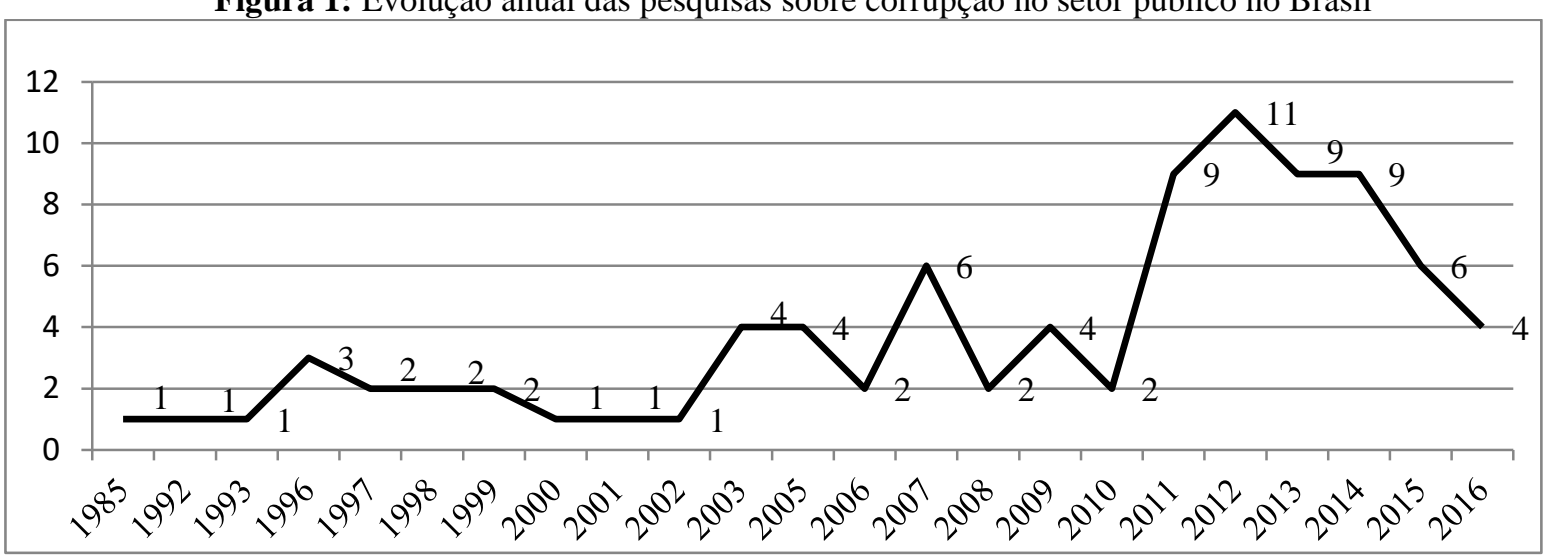

Fonte: dados da pesquisa (2017)

No intuito de identificar e compreender quais as causas da corrupção no Brasil, um dos objetivos deste estudo, procedeu-se à leitura e análise detalhada dos 87 artigos, conforme mencionado na Seção 2, Método de Pesquisa.

Um total de 113 ocorrências de categorias de causas de corrupção foi identificado em 43 artigos de um total de 87 analisados. Nos outros 44 artigos, apesar de estarem aderentes a todos os critérios definidos para esta pesquisa, não foi possível identificar causas da ocorrência da corrupção. Cabe ressaltar, porém, que os 43 artigos que apresentam causas não têm como objetivo estudá-las (ou seja, o mesmo objetivo que este artigo) - as causas foram identificadas por meio da análise e interpretação aprofundada desses artigos e não de mera coleta nos artigos analisados. Assim, e seguindo as etapas descritas na Seção 2, chegou-se a um conjunto de 10 categorias que serão detalhadas a seguir, em ordem decrescente de frequência. A Tabela 1 apresenta as 10 causas da corrupção identificadas, a quantidade de artigos que as abordaram e alguns exemplos de evidências.

Tabela 1: Causas da Corrução no Brasil

\begin{tabular}{|c|c|c|}
\hline Causas & Freq. & Evidências \\
\hline Impunidade & 23 & [...] Em muitos processos, "tudo termina em pizza" [...] \\
\hline 2. Burocratização & 18 & $\begin{array}{l}{[. . .] \text { Muitas vezes o processo é tão demorado e burocrático que }} \\
\text { a propina ocorre para furar a fila [...] }\end{array}$ \\
\hline 3. Fatores Culturais & 15 & $\begin{array}{l}\text { [...] todos fazem assim, não teria problema eu fazer também } \\
\text { (corromper) }[\ldots]\end{array}$ \\
\hline 4. Sistema político corrupto & 13 & $\begin{array}{l}\text { [...] As variedades socioculturais da compra de votos são } \\
\text { muitas [...] }\end{array}$ \\
\hline $\begin{array}{l}\text { 5. Implementação inadequada } \\
\text { de leis anticorrupção }\end{array}$ & 8 & $\begin{array}{l}{[\ldots] \text { Muitas vezes a lei existe, mas não se tem um processo }} \\
\text { efetivo de controle }[\ldots]\end{array}$ \\
\hline $\begin{array}{l}\text { 6. Baixos níveis de } \\
\text { transparência }\end{array}$ & 8 & [...] A transparência diminui a assimetria da informação [...] \\
\hline 7. Setor privado que corrompe & 8 & $\begin{array}{l}\text { [...] A busca de vantagem indevida, para aumentar os ganhos } \\
\text { da empresa privada pode gerar o ato de corrupção no setor } \\
\text { público [...] }\end{array}$ \\
\hline 8. Estado grande e paternalista & 7 & $\begin{array}{l}{[. . .] \text { O estado é muito grande e se torna difícil de controlar. A }} \\
\text { tecnologia e a transparência podem contribuir para disseminar } \\
\text { a informação e aumentar a participação do cidadão [...] }\end{array}$ \\
\hline $\begin{array}{l}\text { 9. Baixo political savvy dos } \\
\text { cidadãos }\end{array}$ & 7 & $\begin{array}{l}{[\ldots] \text { Quanto à instrução, nota-se que, quanto mais anos de }} \\
\text { estudo o indivíduo tem, menor a tolerância em relação ao } \\
\text { "jeitinho" [...] }\end{array}$ \\
\hline 10. Baixos níveis de compliance & 6 & $\begin{array}{l}{[\ldots] \text { deve-se ter regras, e essas regras devem ser }} \\
\text { acompanhadas, controladas e principalmente cumpridas }[\ldots]\end{array}$ \\
\hline TOTAL & 113 & \\
\hline
\end{tabular}

As categorias de causas da corrupção Impunidade, Burocratização e Fatores Culturais, foram os que mais 
apareceram nos artigos analisados, com respectivamente 23, 18 e 15 artigos, que correspondem a quase $40 \%$ das raízes e causas da corrupção.

A expressão "Tudo termina em Pizza" é um símbolo da Impunidade, associada a 23 dos artigos analisados. Essa expressão se aplica a investigações de corrupção perpetradas por servidores públicos ou por políticos que se arrastam por anos, e trazem como consequência poucas sanções ou reprimendas (TAYLOR; BURANELLI, 2007). Outra causa encontrada é a Burocratização, e ocorre quando um burocrata usa indevidamente sua margem de discricionariedade para favorecer seus interesses privados em detrimento do interesse público (GARDINER, 2005). Nessa linha, quanto maior o aparato burocrático maior será o espaço discricionário no qual um servidor público pode atuar de acordo com seus interesses privados. Já os Fatores Culturais abrangem uma gama ampla de manifestações da cultura nacional, tais como o jeitinho brasileiro (FILGUEIRAS, 2009), a malandragem e o entendimento de que quem não leva vantagem é um perdedor (FILGUEIRAS, 2009). Dentro desses fatores está contemplado de forma bem relevante o fator ético.

O Sistema Político e seus altos níveis de corrupção foi outra causa identificada em 13 artigos. Existe um subsistema de reciprocidade e ganho mútuo nas redes exclusivas de corrupção (SANTOS et al., 2014), que envolve o sistema político. Pode envolver tanto a manipulação e a corrupção eleitoral, como a compra de votos (SPECK, 2003), ou caixa dois, desvio de recursos e outros usos indevidos do poder e da posição política. Embora muitas das manifestações dessa causa tenham mais relação com a corrupção política (que não é o foco desta pesquisa), seus desdobramentos tem impactos diretos na dimensão administrativa, em virtude das implicações mútuas entre essas dimensões.

Por outro lado, a criação e principalmente a aplicação de leis é importante para o controle de níveis de corrução. A Implementação inadequada de leis anticorrupção é uma categoria de causas (presente em oito artigos) porque uma condução inadequada da lei faz com que se altere as características da punição, que é uma variável relevante na determinação da atividade criminosa (GICO JR.; DE ALENCAR, 2011). Outra categoria de causas da corrupção é o Baixo nível de Transparência, ou mais especificamente a falta dela, presente em oito artigos. A transparência pode contribuir com a consolidação da democracia, fazendo com que os stakeholders tenham acesso à informação, reduzindo assim as assimetrias que criam desigualdades na representação dos seus interesses (BALAN, 2014). Além disso, a transparência faria com que uma das razões encontradas de corrupção no estudo fosse minimizada, que é o tamanho do Estado - categoria Estado grande e paternalista -, representado em sete artigos analisados. Outra categoria de causas da corrupção, identificada em oito artigos, está relacionada a Iniciativas do Setor privado. Nessa causa, a corrupção no setor público seria resultado das ofertas de propina ou outras vantagens ilícitas e que contrariam o bem comum (GOUVEA; MONTOYA; WALSH, 2013).

Baixos níveis de Political Savvy dos cidadãos estão presentes em sete artigos. Savvy reúne conhecimento e experiência, permitindo a compreensão de determinando fenômeno bem como a projeção de situações relacionadas. Um indivíduo com IT savvy, por exemplo, conhece as Tecnologias da Informação e como aplicálas em diferentes contextos e situações.

Por fim, a categoria de causas da corrupção com menor número de evidências está relacionada a Baixos níveis de compliance. Um processo de compliance rigoroso capaz de detectar, controlar e punir as ações no combate à corrupção é um instrumento importante para a efetividade das ações contra a corrupção.

\subsection{TIC e Corrupção}

Embora exista um grande esforço para diminuir os níveis de corrupção em diversas nações, o número de países com altos níveis de corrupção é significativo. De acordo com o último relatório divulgado pela Transparency International (2016), mais de um terço (1/3) dos países estão classificados com um score abaixo de 50 (em uma escala de 0 a 100), em sua maioria países em desenvolvimento ou em extrema pobreza.

A grande dificuldade no combate à corrupção é a quantidade de componentes e agentes envolvidos em todo o processo (ROSE-ACKERMAN, 1999; SRIVASTAVA; TEO; DEVARAJ, 2016). Para a eficiência desse processo, é necessário ter um controle rigoroso, o qual seja capaz de prevenir, detectar, controlar e punir iniciativas de corrupção. Neste sentido, a TIC surge como uma ferramenta que pode auxiliar nesta tarefa, com a finalidade de aumentar a qualidade das informações e remover ou diminuir as oportunidades de corrupção (HEEKS, 1998).

Visando entender qual pode ser o papel das TICs, uma segunda revisão sistemática da literatura foi realizada para identificar como a TIC tem sido utilizada em diversas atividades relacionadas ao controle da corrupção. Os achados resultaram em 17 artigos, que discorrem sobre o uso de TIC no combate à corrupção, conforme mostra a 
Figura 2, bem como a evolução anual do número de pesquisas.

Figura 1: Evolução anual das pesquisas sobre corrupção no setor público no Brasil

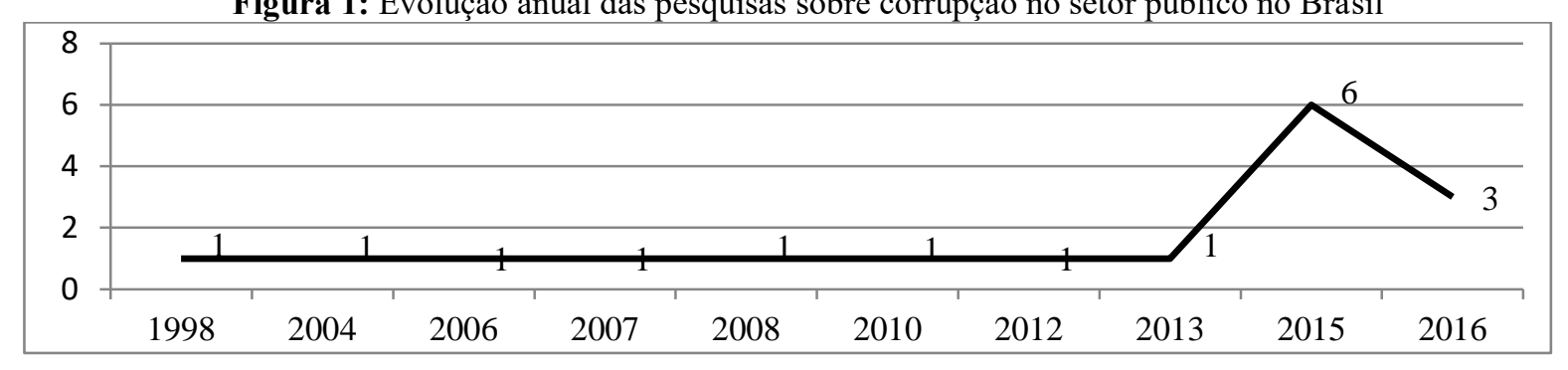

Fonte: dados da pesquisa (2017)

O número de artigos encontrados é pequeno e estes discorrem basicamente sobre as mesmas soluções, quais sejam, o uso da internet para a transparência e a participação do cidadão, mostrando que há muito espaço para a investigação e mesmo utilização das TICs nas iniciativas contra a corrupção. Considerou-se apenas artigos que exploravam soluções baseadas em TICs para fazer frente a problemas de corrupção. Pode-se destacar que nos anos de 2015 e 2016 ocorreu um aumento dos estudos em relação aos outros anos. Os principais resultados são apresentados no Quadro 2.

Quadro 2: Processos utilizados para a redução dos níveis de corrupção

\begin{tabular}{|c|c|c|}
\hline TICs utilizadas & Aplicação e resultados esperados & $\begin{array}{c}\text { Frequên- } \\
\text { cia }\end{array}$ \\
\hline $\begin{array}{l}\text { 1. Mídia eletrônica } \\
\text { e/ou social }\end{array}$ & $\begin{array}{l}\text { A intenção é melhorar a disseminação da informação, aumentando a } \\
\text { divulgação e transparência do desempenho das organizações públicas. }\end{array}$ & 7 \\
\hline $\begin{array}{l}\text { 2. Implantação ou } \\
\text { fortalecimento do atual } \\
\text { Sistema de Informação }\end{array}$ & $\begin{array}{l}\text { Mecanismos de controle administrativo, através de monitoramento. A TIC } \\
\text { como um elemento chave do controle de gestão. Maior eficiência e } \\
\text { transparência para melhorar as práticas burocráticas. Prestação de contas, } \\
\text { participação e procedimentos de trabalho. }\end{array}$ & 6 \\
\hline $\begin{array}{l}\text { 3. Internet/extranet } \\
\text { e/ou portais } \\
\text { governamentais }\end{array}$ & $\begin{array}{l}\text { Busca de processos mais céleres e transparentes, orientando corretamente } \\
\text { as opiniões públicas na Internet, reforçando a credibilidade do governo } \\
\text { para manter a estabilidade social. Na direção de aprofundar a democracia } \\
\text { por meio de processos digitais. }\end{array}$ & 5 \\
\hline 4. Governo Eletrônico & $\begin{array}{l}\text { Busca na melhora do controle e maior participação dos serviços públicos, } \\
\text { além de melhorar a má informação sobre a corrupção. }\end{array}$ & 3 \\
\hline $\begin{array}{l}\text { 5. Business } \\
\text { Intelligence }\end{array}$ & $\begin{array}{l}\text { Informações mais fidedignas, com a redução de erros e eliminação das } \\
\text { redundâncias e permissão de cruzamento e comparação de dados. }\end{array}$ & 2 \\
\hline $\begin{array}{l}\text { 6. Governança } \\
\text { Eletrônica }\end{array}$ & $\begin{array}{l}\text { Governo eletrônico, associado a processos na busca do combate à } \\
\text { corrupção, com a participação do cidadão. }\end{array}$ & 1 \\
\hline TOTAL & & 24 \\
\hline
\end{tabular}

Fonte: dados da pesquisa (2017)

As TICs podem exercer papéis diferenciados na busca da redução dos níveis de corrupção. A partir da análise detalhada dos artigos, foram identificados os papéis de suporte, o papel impulsionador, estratégico, transformador, catalizador e habilitador.

O papel de suporte ocorre muitas vezes através da utilização da internet. O governo e a população podem se beneficiar de sistemas de governo eletrônico (e-gov), que estão a disposição em muitos programas de modernização nas democracias ocidentais (BONSÓN et al., 2012) e tem foco no oferecimento de serviços eletrônicos aos cidadãos. Estes funcionam de forma igual para todos os cidadãos e seguindo regras pré-definidas e auditadas diretamente nos Sistemas de Informação que dão suporte aos serviços eletrônicos. Dessa forma, as possibilidades de solicitações ou oferta de propina (pelos servidores públicos ou por cidadãos, respectivamente) para a obtenção de um serviço ou de algum regalia nesse serviços não são possíveis. As TICs suportam todas as atividades realizadas no âmbito das iniciativas de governo eletrônico.

O papel impulsionador da TIC pode ocorrer, por exemplo, através da disponibilização de dados e informações do governo na busca de um nível elevado de transparência de execução financeira e demais decisões do governo. Esta contribuição pode frear a erosão financeira dos orçamentos e dos sistemas fiscais dos mais diversos Estados soberanos (MELO, 2017). Neste papel as TICs são impulsionadoras porque a transparência off-line pode existir, 
mas ela acaba sendo melhor quando feita com o apoio de TICs.

A TIC pode ter um papel estratégico como instrumento anticorrupção, principalmente quando há uma ligação entre tecnologia e variáveis macroeconômicas, no fornecimento de um governo mais significativo e com capacidade de reduzir a corrupção (IONESCU, 2016). Também como um papel estratégico, Silva e Ralha (2011) propõem utilizar informações das organizações para se criar regras de associação entre empresas concorrentes, para se evitar a corrupção em licitações, principalmente na deteç̧ão de grupos de empresas suspeitas de praticar rodízio em licitações. Neste papel, as TICs permitem uma gestão mais baseada em dados e evidências, permitindo um impacto positivo ainda na origem dos eventos.

Já o papel transformador pode ser explicado pela pesquisa de Belokrylov e Belokrylova (2017) que disserta a respeito do sistema de compras na Rússia, concluindo que a transparência do sistema de contratos públicos, através da tecnologia e a qualificação dos clientes, são fatores determinantes para a eficiência do processo de compras pois permitem o controle social, que contribui para a lisura do processo. Os autores finalizam que a utilização de leilões eletrônicos contribui para transformar a sociedade civil em autoridades competentes em contratos públicos, fiscalizando os processos. Como a relação entre governo e cidadãos ainda não é bidirecional em sua grande parte, o papel da TIC é transformador pela possibilidade de mudar significativamente esse relacionamento.

Lopes (2009, p. 999) ressalta que "a produtividade, a inovação contínua e a mudança tecnológica são consideradas como os principais catalisadores locais do desenvolvimento econômico regional". Como as TICs contribuem significativamente para esta mudança, tem-se o seu papel catalisador, estimulando ou dinamizado as iniciativas. Além do potencial econômico representado pelas TIC, como tecnologia habilitadora de maior competitividade pelo incremento de produtividade que proporciona, outro foco, não menos importante, é o desenvolvimento de negócios baseados em TIC (CUNHA; MIRANDA, 2013). Segundo os autores, as iniciativas de governança eletrônica são essenciais pelo seu potencial de catalisar e habilitar a promoção de práticas democráticas, bem como pelo seu potencial de facilitadoras do relacionamento mais eficiente entre governo e cidadão.

Outro fator identificado nos achados diz respeito à intensidade do ato de corrupção, isto é, a petty corruption e a grand corruption. A primeira é a corrupção que ocorre com mais frequência, todavia, o valor monetário e o impacto envolvido é menor. A grand corruption representa uma ocorrência menor de eventos, porém com valores e impacto mais expressivos. Essas pequenas corrupções podem ocorrer nas tarefas mais simples do cotidiano e por isso aparecem com um número maior nos artigos analisados.

Mesmo que se tenha todo um aparato tecnológico, devem-se levar em consideração outros fatores teóricos, sociais e principalmente individuais (ROSE-ACKERMAN, 1978; MACRAE, 1982; HUSTED, 1994; ANAND et al., 2004). Embora a TIC contribua para a deteç̧ão e a redução dos níveis de corrupção, como demostrado anteriormente, ela pode não ter efeito, ou pior, pode criar um efeito contrário, ou seja, pode oferecer novas oportunidades para a corrupção (HEEK, 1998) caso não tenha a devida transparência e controle realizado por órgãos fiscalizadores (SRIVASTAVA; TEO; DEVARAJ, 2016; FERREIRA et al., 2016; CALDAS; COSTA; PAGLIARUSSI, 2016).

\subsection{Como a TIC contribui para a redução dos níveis de corrupção?}

O resultado da revisão sistemática de literatura apresentou seis aplicações de TIC que podem contribuir com a redução dos níveis de corrupção, quais sejam: Mídia eletrônica e/ou social; Implantação ou fortalecimento do atual Sistema de Informação; Internet/extranet e/ou portais governamentais; Governo Eletrônico; Business Intelligence e Governança Eletrônica.

Visando embasar essa discussão, procedeu-se à análise detalhada das razões para a corrupção juntamente com as publicações que abordam o uso da TIC contra a corrupção em diferentes fatores, causas e níveis. O Quadro 3 contém a distribuição das 10 causas de corrupção no Brasil identificadas previamente (conforme a Tabela 1) associadas com as aplicações identificadas no Quadro 2 de como a TIC pode contribuir para reduzir os níveis de corrupção.

Quadro 3: Contribuição da TIC nas causas da Corrupção no Brasil

\begin{tabular}{|l|l|}
\hline \multicolumn{1}{|c|}{ Causas da corrupção } & $\begin{array}{c}\text { Aplicações de TIC com potencial de suporte à iniciativas contra a } \\
\text { corrupção }\end{array}$ \\
\hline \multirow{2}{*}{ Impunidade } & Mídia eletrônica e/ou social \\
\cline { 2 - 2 } & Business intelligence \\
\hline
\end{tabular}




\begin{tabular}{|c|c|}
\hline Causas da corrupção & $\begin{array}{c}\text { Aplicações de TIC com potencial de suporte à iniciativas contra a } \\
\text { corrupção }\end{array}$ \\
\hline \multirow{4}{*}{ Burocratização } & Mídia eletrônica e/ou social \\
\hline & Implantação ou fortalecimento do Sistema de Informação atual \\
\hline & Internet/extranet e/ou portais governamentais \\
\hline & Governo Eletrônico \\
\hline \multirow{3}{*}{ Fatores Culturais } & Mídia eletrônica e/ou social \\
\hline & Internet/extranet e/ou portais governamentais \\
\hline & Governo Eletrônico \\
\hline \multirow{2}{*}{ Sistema político corrupto } & Business intelligence \\
\hline & Governança Eletrônica \\
\hline $\begin{array}{l}\text { Implementação inadequada de leis } \\
\text { anticorrupção }\end{array}$ & Implantação ou fortalecimento do Sistema de informação atual \\
\hline \multirow{4}{*}{ Baixos níveis de transparência } & Mídia eletrônica e/ou social \\
\hline & Implantação ou fortalecimento do Sistema de informação atual \\
\hline & Internet/extranet e/ou portais governamentais \\
\hline & Governo Eletrônico \\
\hline \multirow{2}{*}{ Setor privado que corrompe } & Business intelligence \\
\hline & Implantação ou fortalecimento do Sistema de informação atual \\
\hline \multirow{2}{*}{ Estado grande e paternalista } & Governo Eletrônico \\
\hline & Governança Eletrônica \\
\hline \multirow{2}{*}{ Baixo political savvy dos cidadãos } & Internet/extranet e/ou portais governamentais \\
\hline & Mídia eletrônica e/ou social \\
\hline \multirow{4}{*}{ Baixos níveis de compliance } & Implantação ou fortalecimento do Sistema de informação atual \\
\hline & Governo Eletrônico \\
\hline & Business intelligence \\
\hline & Governança Eletrônica \\
\hline
\end{tabular}

As TICs podem ajudar na redução dos níveis de corrupção permitindo o rastreamento das atividades dos cidadãos, além de estar monitorando e controlando o comportamento dos funcionários do governo (SHIM; EOM, 2008). Isso pode ocorrer com a implantação de Sistemas de Informação. Novos Sistemas de Informação (SI), melhor desenhados e mais voltados para as reais necessidades das organizações públicas (e não a simples adaptação de SIs desenvolvidos para a iniciativa privada) podem contribuir para reduzir de maneira efetiva a burocratização que ainda impera em muitos espaços no setor público. De fato, não é suficiente apenas automatizar as tarefas da forma como estão hoje, pois o resultado será um processo burocrático automatizado. Faz-se necessária uma análise de quais atividades e documentos são imprescindíveis para a execução de cada processo administrativo. A desburocratização é importante para reduzir o poder discricionário do servidor público, que se manifesta como os graus de liberdade que um servidor público tem para decidir aprovar ou não o pedido de um cidadão. Esses graus de liberdade podem ser usados como uma forma de solicitar propina para que um desfecho favorável ocorra. Segundo Guimarães e Medeiros (2005), novos modelos para o relacionamento Estado/sociedade surgiram com a evolução da informática, dos meios de comunicação e particularmente da Internet. Para Cunha e Miranda (2013), os avanços da TIC e a Internet geraram oportunidades para transformar o relacionamento entre o governo e os cidadãos, contribuindo para alcançar os objetivos da boa governança.

A facilidade do acesso à internet tem gerado iniciativas e demandas que pressionam os governos a inovar em modalidades de relacionamento entre o papel burocrático do governo e os cidadãos (BONSÓN et al., 2012). A utilização da internet para portais governamentais busca processos mais céleres e transparentes, orientando corretamente as opiniões públicas na Internet, reforçando a credibilidade do governo para manter a estabilidade social, na direção de aprofundar a democracia por meio de processos digitais. Todos esses processos contribuem com a desburocratização, além de melhorar os níveis de transparência na busca da redução dos níveis de corrupção.

A utilização das TICs pode aumentar a qualidade das informações e remover ou diminuir as oportunidades de corrupção (HEEKS, 1998). Apesar dessa afirmação de Heeks (1998) ser de certa forma antiga, Srivastava, Teo e Devaraj (2016), colocam que mesmo a tecnologia tendo um grande potencial de transformação e facilitando a transparência nas transações, para se lidar com a corrupção, o assunto ainda está em fase inicial. Pinho (2008) coloca que as TICs têm um enorme potencial democrático, mas para isso fazem-se necessárias a transparência e 
a participação popular. Srivastava, Teo e Devaraj (2016) complementam que o governo eletrônico é uma oportunidade de aumentar a transparência de uma nação, reduzindo a ocorrência das diferentes formas de corrupção.

O baixo nível de transparência pode estar ligado a práticas ilícitas e a falta de controle, mesmo com o esforço para a implantação de novos sistemas informatizados para a socialização e transparência dos dados. Neste sentido, mecanismos de governança eletrônica e a transparência, com o auxílio da tecnologia, surgem como uma poderosa ferramenta que podem aumentar a qualidade das informações e remover ou diminuir as oportunidades de corrupção (HEEKS, 2000).

Dispositivos de TIC são fundamentais para que essa transparência ocorra, seja por meio de portais, aplicativos ou mídias sociais que permitam ao cidadão acompanhar as ações da administração pública em nível municipal, estadual ou nacional, e exercer o controle social. Com baixo nível de transparência, o cidadão não consegue acompanhar os atos da administração pública, e assim, um importante direito de participação é negado a esse cidadão.

No caso da transparência, a TIC tem um papel habilitador, já que é praticamente impossível promover transparência e levar a informação ao cidadão sem níveis razoáveis de TIC. O mesmo ocorre com a desburocratização, causa da corrupção na qual a TIC também tem um papel habilitador. Menores níveis de burocracia demandam sistemas inteligentes, acessados de diferentes dispositivos (computadores, tablets, smartphones, e mesmo dispositivos inteligentes em espaços públicos, por meio da Internet das Coisas). Já no tocante à compliance, a TIC tem um papel de suporte, principalmente com a utilização de Business Intelligence, considerando que ela não é fundamental para estabelecer programas e controles de compliance, muito embora agregue valor por meio do seu uso, principalmente porque gera informações mais fidedignas, com a redução de erros, eliminação das redundâncias, permissão de cruzamento e comparação de dados (REIS et al., 2015).

Essa comparação de dados gera informações que podem ser utilizadas por todos na busca da redução dos níveis de corrupção. Porém, para Klitgaard (1988) a corrupção é um problema de informação assimétrica e incentivo, o que pode ser explicado pelo modelo principal-agente-cliente descrito na Teoria da Agência. De acordo com este modelo, os funcionários públicos são honestos, mas caso tenham a um monopólio e poder discricionário na sua administração e com a falta de prestação de contas, a corrupção poderá ocorrer. Ou seja, o problema da corrupção surge em situações em que existe um problema de assimetria de informações, em que os agentes sabem muito mais sobre a administração de qualquer um dos princípios ou os clientes (KRISHNAN; TEO; LIM, 2013).

Considerando todas as causas e os papéis da TIC encontradas a respeito da corrupção, as iniciativas concretas para a redução dos níveis de corrupção no Brasil devem crescer nos próximos anos. Algumas iniciativas no próprio país e também em outros países. Exemplos como os relatados por Young (2004) acerca da atuação do Instituto Ethos, o Movimento Contra a Corrupção (CONTRA CORRUPÇÃO, 2017), as iniciativas do Ministério Público Federal (COMBATE À CORRUPÇÃO, 2017) e do Portal Transparência.org (TRANSPARENCIA, 2016). Internacionalmente, cabe destacar o projeto Open, em Singapura (KIM; KIM; LEE, 2009), os esforços do Accountability Lab, com atuação nos Estados Unidos, Nepal e Liberia (ACCOUNTABILITY, 2017), o Anti Corruption Authorities (ACAUTHOTITIES, 2015), ligado ao Banco Mundial, as diversas iniciativas Uruguaias relatadas por Solares (2010) e o Fórum Nacional Anticorrupção (NACF, 2017), na África do Sul. Meagher (2005) analisou o papel de 14 agências governamentais contra a corrupção, listando diversos casos de sucesso. O grupo U4 Partners, que envolve nove países (Austrália, Alemanha, Bélgica, Dinamarca, Reino Unido, Finlândia, Suécia e Suíça) também relata esforços e inciativas de sucesso contra a corrupção nos países envolvidos (U4PARTNERS, 2015). Bertot, Jaeger, Grimes (2010) descrevem uma série de inciativas em países como Chile, Ilhas Fiji, Estados Unidos da América, Índia, Paquistão e Filipinas.

\section{Considerações Finais}

A corrupção é um fator que pode alterar a qualidade de vida da sua população, afetando o desenvolvimento social de um país. Sendo um problema grave e complexo, todo tipo de recursos precisa ser utilizada para tornar as resultados mais efetivos. Neste sentido, este estudo teve como principal objetivo identificar ações de TICs voltadas ao combate da corrupção, bem como as causas da corrupção no Brasil segundo pesquisas anteriores e identificadas por meio de um levantamento sistemático da literatura, o que possibilitou a análise dessas causas à luz do potencial de aplicação das TICs. Como resultado, foram cruzadas as informações para discutir qual pode ser o papel da TIC na redução dos níveis da corrupção. Os principais papéis encontrados foram: suporte, impulsionador, estratégico, transformador, catalizador e habilitador. 
O estudo revelou que a impunidade é um dos principais motivadores da corrução. Logo em seguida vieram os processos e procedimentos excessivamente burocráticos. Quanto à burocratização, as TIC tem um bom potencial de contribuição, pois possibilitam a automação de tarefas e a redução das tarefas manuais. Os autores identificaram ainda que nas raízes e razões da corrupção no Brasil encontram-se questões relativas à falta de responsabilização, bem como os aspectos culturais das instituições públicas.

Os resultados da revisão sistemática de literatura mostram seis principais aplicações de TICs, quais sejam: Mídia eletrônicas e/ou social; Implantação ou fortalecimento do atual Sistema de Informação; Internet/extranet e/ou portais governamentais; Governo Eletrônico; Business Intelligence e Governança Eletrônica. Todas essas aplicações buscam principalmente a disseminação da informação, a participação do cidadão, o controle social e a Segurança da Informação na prestação de serviços ao cidadão. Essas aplicações representam abordagens indiretas à corrupção, mas mesmo assim podem ser úteis na redução de vulnerabilidades à brechas que podem gerar corrupção.

Quando associadas as causas da corrupção às aplicações de TIC, percebe-se que até o momento a utilização de mídias sociais e consequentemente da Internet tem se mostrado as principais ferramentas de TIC utilizadas para melhorar a transparência, a impunidade e também a burocratização. Outra aplicação muito citada nos artigos foi a utilização de Sistemas de Informação melhor adaptados às necessidades e características do setor público (ao invés de uma simples adaptação daqueles desenvolvidos para o setor privado) para melhorar os processos e diminuir a burocratização, ampliar o controle e a compliance e consequentemente diminuir a impunidade através da apresentação dos seus resultados. Desta forma, o estudo identificou que em termos de fatores administrativos estruturais a TIC pode ter um papel habilitador para as causas, Falta de Transparência e Burocratização, de forma a ampliar a transparência e aumentar a agilidade na gestão pública para reduzir as instâncias e processos burocráticos, bem como o papel de suporte, porque gera informações mais fidedignas com a redução de erros e a eliminação das redundâncias.

A principal contribuição teórica do artigo é relacionar as aplicações de TIC com as causas da corrupção, embasando a discussão de como a utilização da TIC pode atuar para evitar ou atenuar os impactos da corrupção. Como contribuição prática deste estudo, destaca-se a possibilidade dos órgãos governamentais e reguladores definirem meios que mitiguem os impactos e aplicações de TIC identificadas, no intuito de diminuir os níveis de corrupção no Brasil. Os resultados desta pesquisa devem ser compreendidos dentro dos limites do contexto estudado.

A continuidade deste estudo envolve a realização de um estudo comparativo das causas da corrupção entre países com menor e maior nível de corrupção, verificando as semelhanças e diferenças. Envolve também a realização de uma metanálise e a proposição de um modelo de causa e efeito entre os itens encontrados e a corrupção, para contribuir com a avaliação do efeito moderador dos fatores culturais e comportamentais no nível de corrupção. Adicionalmente, estudos de caso podem contribuir no entendimento pontual da utilização de aplicações de TIC visando reduzir a ocorrência da corrupção, bem como a identificação de outros tipos de papéis desempenhados pela TIC.

\section{Referências}

ACAUTHORITIES. Anticorruption Authorities. Disponível em: $<$ https://www.acauthorities. org/>. Acesso em 26 de maio de 2017.

ACCOUNTABILITY. Accountability Lab. Disponível em: <http://www.accountabilitylab. org/>. Acesso em 30 de maio de 2017

AGUNE, R.; CARLOS, J. Governo eletrônico e novos processos de trabalho. Gestão pública no Brasil contemporâneo. São Paulo: Fundap, p. 302-315, 2005.

AIDT, Toke S. Economic analysis of corruption: a survey. The Economic Journal, v. 113, n. 491, p. F632-F652, 2003.

ALADWANI, Adel M. Corruption as a source of e-Government projects failure in developing countries: A theoretical exposition. International Journal of Information Management, v. 36, n. 1, p. 105-112, 2016.

ANAND, Vikas; ASHFORTH, Blake E.; JOSHI, Mahendra. Business as usual: The acceptance and perpetuation of corruption in organizations. The Academy of Management Executive, v. 18, n. 2, p. 39-53, 2004.

BALÁN, Manuel. Assessing Progress in Transparency and Anticorruption. Latin American Research Review, v. 49, n. 2, p. 262-272, 2014. 
BARDIN, Lawrence. Análise de conteúdo. Lisboa: edições, v. 70, p. 225, 197

BELOKRYLOV, Kirill A.; BELOKRYLOVA, Olga S. Transparency of the governmental procurement for civil society and the factors of their efficiency. Revista ESPACIOS, v. 38, n. 31, 2017.

BERTOT, John C.; JAEGER, Paul T.; GRIMES, Justin M. Using ICTs to create a culture of transparency: Egovernment and social media as openness and anti-corruption tools for societies. Government information quarterly, v. 27, n. 3, p. 264-271, 2010.

BONIFÁCIO, Robert. A afeição dos cidadãos pelos políticos mal-afamados: identificando os perfis associados à aceitação do 'rouba, mas faz' no Brasil. Opinião Pública, v. 19, n. 2, p. 320-345, 2013.

BONSÓN, Enrique et al. Local e-government 2.0: Social media and corporate transparency in municipalities. Government information quarterly, v. 29, n. 2, p. 123-132, 2012.

BRERETON, Pearl et al. Lessons from applying the systematic literature review process within the software engineering domain. Journal of systems and software, v. 80, n. 4, p. 571-583, 2007.

BRESSER PEREIRA, Luiz Carlos. Reforma gerencial do Estado, teoria política e ensino da administração pública. Revista Gestão \& Políticas Públicas, v. 1, n. 2, 2012.

BROL, Marcin. Institutional determinants of corruption. Ekonomia i Prawo, v. 15, n. 1, p. 21, 2016.

CALDAS, Olavo Venturim; COSTA, Cristiano Machado; PAGLIARUSSI, Marcelo Sanches. Corrupção e composição dos gastos governamentais: evidências a partir do Programa de Fiscalização por Sorteios Públicos da Controladoria-Geral da União. Revista de Administração Pública, v. 50, n. 2, p. 237-264, 2016.

CARRARO, A. et al. Formação de empresas e corrupção: uma análise para os estados brasileiros. Encontro de Economia da Região Sul-ANPEC/SUL, 2011.

COMBATE A CORRUPÇÃO. Ministério Público Federal. Disponível em: <http//www. combateacorrupcao.mpf.mp.br/>. Acesso em 30 de maio de 2017.

CONTRA CORRUPÇÃO. Movimento Contra a Corrupção. Disponível em: <http://www. contracorrupcao.org/> . Acesso em 26 de maio de 2017

COOPER, Harris; HEDGES, Larry V.; VALENTINE, Jeffrey C. (Ed.). The handbook of research synthesis and meta-analysis. Russell Sage Foundation, 2009.

CUNHA, Maria Alexandra Viegas Cortez da; MIRANDA, Paulo Roberto de Mello. O uso de TIC pelos governos: uma proposta de agenda de pesquisa a partir da produção acadêmica e da prática nacional. Organizações \& Sociedade, v. 20, n. 66, p. 543-566, 2013.

DEL MONTE, Alfredo; PAPAGNI, Erasmo. Public expenditure, corruption, and economic growth: the case of Italy. European journal of political economy, v. 17, n. 1, p. 1-16, 2001.

DIAS, Joilson; BENTO, Felipe. Corrupção e teoria econômica. Revista Economia \& Tecnologia, v. 7, n. 3, 2011.

DREHER, Axel; GASSEBNER, Martin. Greasing the wheels? The impact of regulations and corruption on firm entry. Public Choice, p. 1-20, 2013.

FERLIE, E. (1996). The new public management in action. Oxford University Press, USA.

FERRAZ, Claudio; FINAN, Frederico. Exposing corrupt politicians: the effects of Brazil's publicly released audits on electoral outcomes. The Quarterly Journal of Economics, v. 123, n. 2, p. 703-745, 2008.

FERREIRA, Manuel Portugal; CARREIRA, Helder Costa; LI, Dan; SERRA, Fernando Ribeiro. O Efeito Moderador da Corrupção do País de Origem Sobre a Capacidade do País Receptor de Atrair IED. Brazilian Business Review, v. 13, n. 4, p. 98, 2016.

FILGUEIRAS, Fernando. A tolerância à corrupção no Brasil: uma antinomia entre normas morais e prática social. Opinião Pública, v. 15, n. 2, p. 386-421, 2009.

FILGUEIRAS, Fernando; ARANHA, Ana Luiza Melo. Controle da corrupção e burocracia da linha de frente: regras, discricionariedade e reformas no Brasil. Revista Dados, v. 54, n. 1, 2011.

FOX, Jonathan. The uncertain relationship between transparency and accountability. Development in practice, v. 17, n. 4-5, p. 663-671, 2007.

GARCIA, Ricardo Letizia. A economia da corrupção: teoria e evidências: uma aplicação ao setor de obras 
rodoviárias no Rio Grande do Sul. 2003.

GARDINER, John. Defining corruption. Political corruption: Concepts and contexts, v. 3, p. 25-40, 2002.

GICO JR, Ivo T.; DE ALENCAR, Carlos HR. Corrupção e judiciário: a (in) eficácia do sistema judicial no combate à corrupção. Revista Direito GV, v. 7, n. 1, 2011.

GOUVEA, Raul; MONTOYA, Manuel; WALSH, Steve. How the Corruption Quadruple Helix Affects BRIC: A Case Study of Corruption in Big Emerging Economies. J. Pol. \& L., v. 6, p. 1, 2013.

GUIMARÃES, Tomás de Aquino; MEDEIROS, Paulo Henrique Ramos. A relação entre governo eletrônico e governança eletrônica no governo federal brasileiro. Cadernos Ebape. Br, v. 3, n. 4, p. 01-18, 2005.

HABIB, Mohsin; ZURAWICKI, Leon. Corruption and foreign direct investment. Journal of international business studies, v. 33, n. 2, p. 291-307, 2002.

HEEKS, Richard. Government data: Understanding the barriers to citizen access and use. Institute for Development Policy and Management, University of Manchester, 1998.

HERZFELD, Thomas; WEISS, Christoph. Corruption and legal (in) effectiveness: an empirical investigation. European Journal of Political Economy, v. 19, n. 3, p. 621-632, 2003.

HUSTED, Bryan W. Honor among thieves: A transaction-cost interpretation of corruption in third world countries. Business Ethics Quarterly, v. 4, n. 1, p. 17-27, 1994

IONESCU, Luminita. E-government and social media as effective tools in controlling corruption in public administration. Economics, Management, and Financial Markets, v. 11, n. 1, p. 66-72, 2016.

JAEGER, Paul T.; BERTOT, John Carlo. Transparency and technological change: Ensuring equal and sustained public access to government information. Government Information Quarterly, v. 27, n. 4, p. 371-376, 2010.

JAIN, Arvind K. Corruption: A review. Journal of economic surveys, v. 15, n. 1, p. 71-121, 2001

KIM, Seongcheol; KIM, Hyun Jeong; LEE, Heejin. An institutional analysis of an e-government system for anticorruption: The case of OPEN. Government Information Quarterly, v. 26, n. 1, p. 42-50, 2009.

KITCHENHAM, Barbara. Procedures for performing systematic reviews. Keele, UK, Keele University, v. 33, n. 2004, p. 1-26, 2004.

KLITGAARD, Robert. Controlling corruption. Univ of California Press, 1988.

KRISHNAN, Satish; TEO, Thompson SH; LIM, Vivien KG. Examining the relationships among e-government maturity, corruption, economic prosperity and environmental degradation: A cross-country analysis. Information \& Management, v. 50, n. 8, p. 638-649, 2013.

KÜHL TELES, Vladimir. Institutional quality and endogenous economic growth. Journal of Economic Studies, v. 34, n. 1, p. 29-41, 2007.

LOPES, Maria do Céu Baptista. Redes, tecnologia e desenvolvimento territorial. In: Congresso de Desenvolvimento Regional de Cabo Verde: Redes de Desenvolvimento Regional. 2009. p. 995-1015.

MACRAE, John. Underdevelopment and the economics of corruption: A game theory approach. World Development, v. 10, n. 8, p. 677-687, 1982.

MALHOTRA, Naresh K. Pesquisa de marketing: uma orientação aplicada. Bookman Editora, 2012.

MEAGHER, Patrick. Anti-corruption agencies: Rhetoric Versus reality. The Journal of Policy Reform, v. 8, n. 1, p. 69-103, 2005.

MELO, Felipe Luiz; SAMPAIO, Luciano; OLIVEIRA, Renato. Corrupção Burocrática e Empreendedorismo: Uma Análise Empírica dos Estados Brasileiros. RAC-Revista de Administração Contemporânea, v. 19, n. 3, 2015.

MELO, Luciana Grassano. INTERCÂMBIO DE INFORMAÇÃO CONTRA A FRAUDE E EVASÃO FISCAIS. Revista Jurídica, v. 2, n. 47, p. 159-173, 2017.

MENDES BORINI, Felipe; CORREA GRISI, Fernando. A corrupção no ambiente de negócios: survey com as micro e pequenas empresas da cidade de São Paulo. Revista de Administração-RAUSP, v. 44, n. 2, 2009.

NACF. NATIONAL ANTI-CORRUPTION FORUM. Disponível em: $<$ http://www.nacf.org.za/http://www.accountabilitylab.org/>. Acesso em 30 de maio de 2017 
PASCARELLI FILHO, Mario. A nova administração pública: profissionalização, eficiência e governança. DVS Editora, 2013.

PEREIRA, José Matias. Reforma do Estado e controle da corrupção no Brasil. Revista de Administração Mackenzie, v. 4, n. 1, 2008.

PINHO, José Antonio Gomes de. Investigando portais de governo eletrônico de estados no Brasil: muita tecnologia, pouca democracia. 2008.

POWER, Timothy J.; GONZALEZ, Júlio. Cultura política, capital social e percepções sobre corrupção: uma investigação quantitativa em nível mundial. Revista de Sociologia e Política, n. 21, 2003.

REIS, A. F.; DACORSO, A. L. R.; TENÓRIO, F. A. G. Influência do uso de Tecnologias de Informação e Comunicação na prestação de contas públicas municipais: um estudo de caso no Tribunal de Contas dos Municípios do Estado da Bahia. Revista de Administração Pública, Rio de Janeiro, v. 49, n. 1, p. 231-51, 2015.

ROSE-ACKERMAN, Susan. Corruption and government: Causes, consequences and reform. Cambridge, UK. Cambridge University Press, 1999.

ROSE-ACKERMAN, Susan. Corruption: A Study in Political Economy, New York: Academic Press, 1978.

SANTOS, Luiz Alberto; COSTA, Paulo Mauricio Teixeira. The contribution of lobby regulation initiatives in addressing political corruption in Latin America. Journal of Public Affairs, v. 14, n. 3-4, p. 379-391, 2014.

SHIM, Dong Chul; EOM, Tae Ho. E-government and anti-corruption: Empirical analysis of international data. Intl Journal of Public Administration, v. 31, n. 3, p. 298-316, 2008.

SILVA, Carlos Vinícius Sarmento; RALHA, Célia Ghedini. Detecção de cartéis em licitações públicas com agentes de mineração de dados. Revista Eletrônica de Sistemas de Informação, Curitiba, v. 10, n. 1, 2011.

SOLARES, Angélica Pulido. Uruguay: A Case of Success in Latin America. In: Unpublished paper prepared for the seminar "Transitions to Good Governance" at the Hertie School of Governance, Fall Semester. 2010 .

SPECK, Bruno Wilhelm. A compra de votos: uma aproximação empírica. Opinião Pública, v. 9, n. 1, p. 148$169,2003$.

SRIVASTAVA, Shirish C.; TEO, Thompson SH; DEVARAJ, Sarv. You Can't Bribe a Computer: Dealing with the Societal Challenge of Corruption Through ICT. Mis Quarterly, v. 40, n. 2, p. 511-526, 2016.

TANZI, Vito; DAVOODI, Hamid. Corruption, public investment, and growth. IMF Working Paper, 1997. Disponível em: <www.imf.org/external/pubs/ft/wp/wp97139.pdf>. Acesso em: 23 set. 2016.

TAYLOR, Matthew M.; BURANELLI, Vinícius C. Ending up in pizza: accountability as a problem of institutional arrangement in Brazil. Latin American Politics and Society, v. 49, n. 1, p. 59-87, 2007.

TRANSPARENCY. (2016). TRANSPARENCY INTERNATIONAL. Disponível em: >https://www.transparency.org/country\#BRA_DataResearch>. Acesso em: 20 de fev. 2017.

U4. U4 Anti-Corruption Resource Centre. Disponível em: $<$ http://www.u4.no/http://www. accountabilitylab.org/>. Acesso em 16 de março de 2017.

UNODC (United Nations Office on Drugs and Crime). The global programme against corruption - UN anticorruption toolkit, 3rd ed. Vienna: UNODC, 2004.

YILDIZ, Mete. E-government research: Reviewing the literature, limitations, and ways forward. Government information quarterly, v. 24, n. 3, p. 646-665, 2007.

YOUNG, R. Case Story: The Brazilian Programme for Promoting Integrity and the Fight against Corruption. In.: UNODC (United Nations Office on Drugs and Crime). The global programme against corruption - UN anticorruption toolkit, 3rd ed. Vienna: UNODC, 2004. 